REVIEW ARTICLE

S. Vattoth

R. Shah

J.K. Curé

\title{
A Compartment-Based Approach for the Imaging Evaluation of Tinnitus
}

SUMMARY: Tinnitus affects $10 \%$ of the US general population and is a common indication for imaging studies. We describe a sequential compartment-based diagnostic approach, which simplifies the interpretation of imaging studies in patients with tinnitus. The choice of the initial imaging technique depends on the type of tinnitus, associated symptoms, and examination findings. Familiarity with the pathophysiologic mechanisms of tinnitus and the imaging findings is a prerequisite for a tailored diagnostic approach by the radiologist.

ABBREVIATIONS $\mathrm{AV}=$ arteriovenous; $\mathrm{AVF}=$ arteriovenous fistula; $\mathrm{AVM}=$ arteriovenous malformation; $\mathrm{CPA}=$ cerebellopontine angle; $\mathrm{DSA}=$ digital subtraction angiography; $\mathrm{IAC}=$ internal auditory canal; ICA = internal carotid artery; $I \mathrm{H}=$ idiopathic intracranial hypertension; $\mathrm{MD}=$ Ménière disease; NSAIDs = nonsteroidal anti-inflammatory drugs; PSA = persistent stapedial artery; TMJ = temporomandibular joint.

T innitus is defined as an auditory perception of internal origin, usually localized and rarely heard by others. ${ }^{1}$ It is a common presenting symptom, affecting up to $10 \%$ of the US general population. It is most prevalent between 40 and 70 years of age, has a roughly equal prevalence in men and women, and can occur in children. ${ }^{2}$ Tinnitus may be classified as subjective versus objective and pulsatile versus continuous. Pulsatile tinnitus is a discrete repetitive sound that is synchronous with the patient's pulse. Continuous (or nonpulsatile) tinnitus refers to all other rhythms, usually a constant unrelenting noise. Objective tinnitus is audible to the examining/ auscultating physician, whereas subjective tinnitus can only be perceived by the patient. Nonpulsatile tinnitus is almost always subjective, whereas pulsatile tinnitus can be subjective or objective.

\section{Role of Imaging in the Clinical Evaluation of Tinnitus}

Although audiometric evaluation of patients may occasionally identify a source of tinnitus such as otosclerosis, MD, or noiseinduced hearing loss, ${ }^{3}$ most patients with disabling symptoms will eventually require radiologic evaluation. However, even in the most carefully selected cohorts, many patients will have no radiologic abnormalities to explain their tinnitus. On the other hand, many radiologic findings associated with tinnitus are also seen frequently in asymptomatic individuals or occasionally may even occur contralateral to the side of symptoms. These observations are consistent with the current pathophysiologic models of tinnitus, which emphasize the importance of functional abnormalities in the brain stem processing of normal sounds over discrete anatomic lesions. ${ }^{4}$

From the Department of Radiology, Division of Neuroradiology, University of Alabama at Birmingham, Birmingham, Alabama.

S. Vattoth and R. Shah are co-first authors.

Please address correspondence to Surjith Vattoth, MD, DMRD, DNB, FRCR, Department of Radiology, University of Alabama at Birmingham, 619 19th St S, WP 150, Birmingham, AL 35233; e-mail: drsurjith@yahoo.co.uk

Indicates article with supplemental on-line tables.

Indicates open access to non-subscribers at www.ajnr.org

DOI 10.3174/ajnr.A1704

\section{Choice of Imaging Technique and Expected Yield}

Nonpulsatile tinnitus is associated most frequently with functional injuries from exposure to environmental noise or the toxicity of certain medications. Consequently, most patients with isolated nonpulsatile tinnitus do not have imaging abnormalities. ${ }^{5}$ The imaging work-up should be guided by associated symptoms, such as hearing loss, dizziness, or headache. A contrastenhanced MR imaging remains the study of choice for these patients. $^{5}$

In patients with pulsatile tinnitus, nearly $57 \%-100 \%$ of all examinations will reveal imaging abnormalities, ${ }^{6-9}$ though the diagnostic relevance of some imaging findings such as jugular bulb variants is controversial. Some authors consider the presence of these abnormalities as a "positive scan," 8 whereas others regard these findings as incidental because they are relatively common in the general population and are usually asymptomatic. ${ }^{6}$ If these variants are excluded, the yield of imaging is lowered by approximately $20 \%-30 \%{ }^{8}$

There is a general consensus that a contrast-enhanced highresolution $\mathrm{CT}$ of the temporal bone should be the initial imaging study for pulsatile tinnitus. ${ }^{10-12}$ If CT findings are negative, further investigations may still yield abnormalities in $30 \%-40 \%$ of patients. ${ }^{6,7}$ These include carotid sonography to exclude stenosis $^{1}$; contrast-enhanced neck CT angiography to exclude carotid stenosis, dissection, mass, or a compressive lesion in the neck or superior mediastinum ${ }^{2}$; and conventional angiography to rule out a dural AVM, AVF, and other vascular abnormalities of the extracranial carotid arteries. ${ }^{3,6,13}$ Contemporary MR angiography protocols include time-resolved imaging of contrast kinetics, ${ }^{14,15}$ a technique that can demonstrate most vascular lesions without the risks of conventional angiography.

\section{A Compartment-Based Approach to Identify the Etiology of Tinnitus}

A list of various common and uncommon causes of tinnitus is provided in On-line Table 1. In the following pages, we have categorized different conditions that can cause tinnitus according to the anatomic location of the most conspicuous imaging abnormality. A systematic approach in the evaluation of tinnitus not only facilitates a logically organized search for abnormalities but also helps in careful exclusion of pertinent 


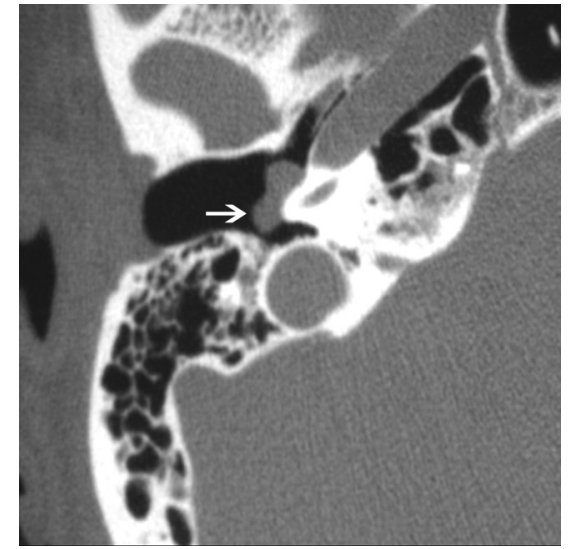

Fig 1. Glomus tympanicum (arrow) in a 30-year-old woman with right-sided pulsatile tinnitus. Axial CT image demonstrates lobulated soft-tissue attenuation in the middle ear overlying the cochlear promontory.

differential diagnoses once an abnormality is identified. A summary of major causes categorized by anatomic location is listed in On-line Table 2.

\section{Scalp}

Extracranial AVMs can rarely cause pulsatile tinnitus. ${ }^{16}$ An enlarged external carotid artery branch may be an important clue to an associated intracranial AVM or dural AVF fed by its branches. ${ }^{17}$ Manual compression of an artery that was seen as mildly enlarged on imaging may suppress or even completely eliminate tinnitus, thereby localizing the origin of the symptoms.

\section{Middle Ear}

The differential diagnosis of middle ear masses that may present with tinnitus includes glomus tympanicum, glomus jugulotympanicum, aberrant or dehiscent ICA, jugular bulb variants, and, rarely, cavernous hemangioma of the middle ear. Radiologic findings associated with persistence of the stapedial artery are included in the section on skull base foramina.

Glomus tumors are vascular paragangliomas located in close proximity to cranial nerves. Glomus tympanicum, the most common tumor of the middle ear cavity, usually arises along the course of the tympanic nerve (Jacobson nerve). Glomus tumors are most frequently located on the cochlear promontory (Fig 1) but can arise almost anywhere in the medial mesotympanum. ${ }^{18}$ The typical appearance of a glomus tumor on temporal bone CT is a round mass based on the cochlear promontory. On MR imaging, these tumors show intense enhancement. Glomus tympanicum usually does not cause erosion of the underlying bone; if the floor of the middle ear cavity is eroded, a glomus jugulotympanicum should be considered (described in the section on jugular foramen masses). Unlike paragangliomas elsewhere in the body, glomus tumors are usually solitary, and the detection of such a lesion does not indicate a need for further imaging to search for additional tumors.

Aberrant ICA. The etiology of aberrant ICA is hypothesized to be an abnormal regression of the cervical ICA during embryogenesis. The inferior tympanic artery, a branch of the ascending pharyngeal artery, undergoes compensatory en- largement and anastomoses with the caroticotympanic artery in the middle ear and then resumes the usual course of the ICA in the petrous part of the carotid canal.

On axial CT images, an aberrant ICA is seen as a tubular structure running horizontally through the middle ear from posterior to anterior (Fig 2A). The inferior tympanic canaliculus is enlarged, and the vertical segment of the petrous ICA is absent. The aberrant ICA is often smaller than the contralateral normal ICA ${ }^{19}$ and may be difficult to detect on conventional MR imaging. On MR angiography, the aberrant ICA extends more laterally than its normal course. This has been termed the 7 or reversed- 7 sign (Fig $2 B) .^{20}$

Dehiscent (Lateralized) ICA. An ICA with a normal course may have a focally dehiscent carotid canal, though this is less common than dehiscence in the setting of an aberrant ICA. The genu of the vertical and horizontal petrous ICA segments lies more laterally and posteriorly, with associated lateral wall dehiscence. The dehiscence is usually near the basal turn of the cochlea.

Jugular Bulb Variants. Dehiscence of the jugular bulb is a normal venous variant, in which a superolateral extension of the jugular bulb extends into the middle ear cavity through a dehiscent sigmoid plate. Otoscopically, this is seen as a blue mass behind an intact tympanic membrane, which may become distended on Valsalva or ipsilateral jugular compression.

A jugular bulb diverticulum is defined as a focal polypoid extension of the jugular bulb superiorly (or, less commonly medially, anteriorly or posteriorly) into the deep temporal bone just behind the IAC, with an intact sigmoid plate. A "high-riding jugular bulb" is defined as an extension of the most cephalad portion of the jugular bulb superior to the floor of the IAC.

Although jugular bulb variants have been associated with tinnitus, ${ }^{21}$ the causal association of these variants with tinnitus remains controversial. ${ }^{6,8,12}$ When one of these variants is found in a patient with pulsatile tinnitus, a search for other treatable causes should still be made.

Cavernous Hemangioma. Cavernous hemangioma is a rare benign neoplasm of the middle ear, which can rarely cause tinnitus and mimic a glomus tympanicum clinically, radiographically, and otoscopically. ${ }^{22}$ Middle ear hemangiomas should be differentiated from the more common geniculate ganglion hemangiomas, which usually present with facial nerve palsy ${ }^{23}$ but may rarely cause pulsatile tinnitus. ${ }^{24,25}$ The diagnosis is often possible only at surgery.

Temporal Bone. Several temporal bone lesions have been associated with tinnitus. These have the following classifications.

\section{Abnormal Lucency or Sclerosis}

Otosclerosis. Otosclerosis or otospongiosis is an idiopathic infiltrative process of the petrous temporal bone that may present with tinnitus. The most common type is fenestral otosclerosis $(85 \%)$, which presents with conductive hearing loss. Cochlear or retrofenestral otosclerosis (15\%) causes additional sensorineural hearing loss. Chronic subjective tinnitus is a common feature of clinical otosclerosis, reported in $\leq 65 \%$ of patients. ${ }^{26}$

In early stages of otosclerosis, a radiolucent focus may be 

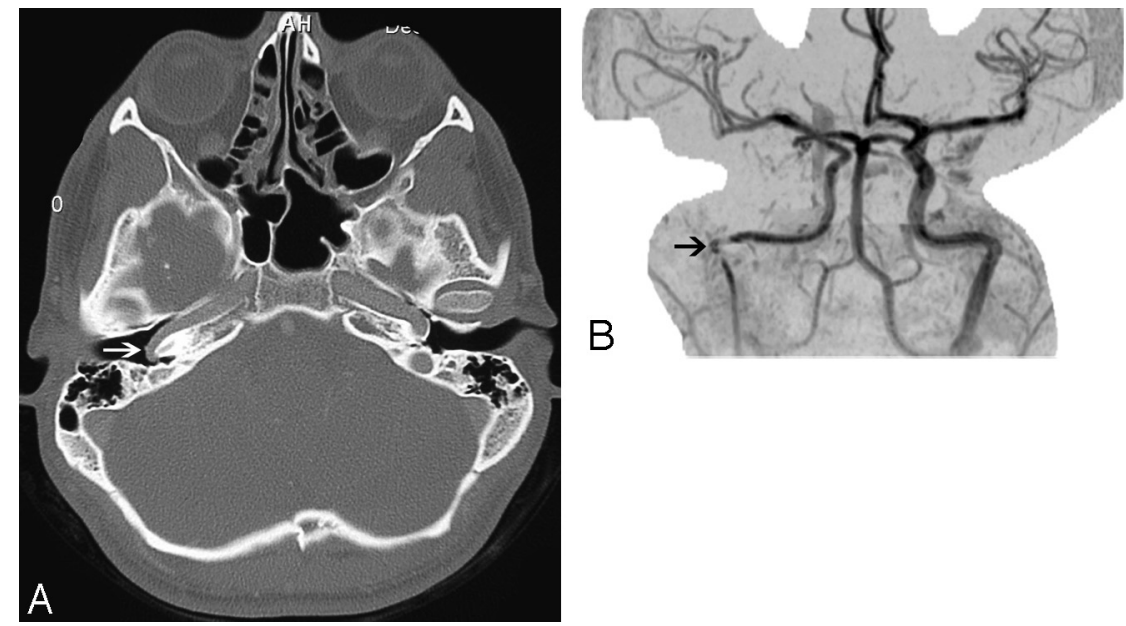

Fig 2. Aberrant course of the ICA in a 25 -year-old man presenting with pulsatile tinnitus. $A$, Enhanced axial CT image demonstrates an abnormal lateral course of the right ICA through the middle ear (white arrow). Also note dehiscence of the overlying bony plate. $B$, Anteroposterior projection image from the MR angiogram of the same patient demonstrates decreased caliber and lateral deviation of the aberrant ICA on the "right reversed-7 sign" (black arrow).

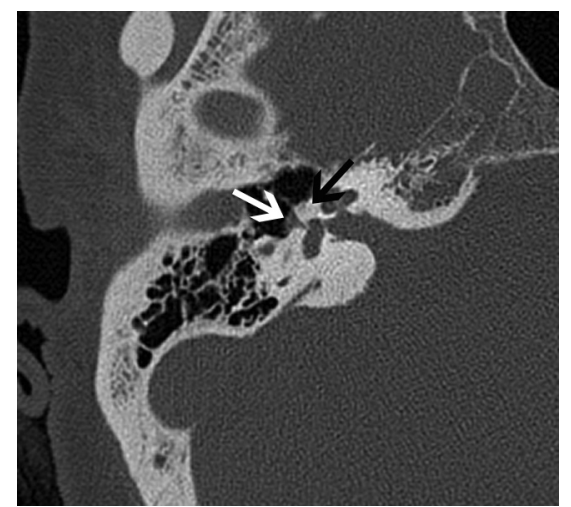

Fig 3. Fenestral otospongiosis in a 35-year-old woman with hearing loss and tinnitus. Axial CT image demonstrates soft-tissue attenuation in the oval window (white arrow) and abnormal lucency at the fissula antefenestram (black arrow).

seen at the fissula ante fenestram, which is a cleft of fibrocartilaginous tissue located just anterior to the oval window. As the disease progresses, this lucency involves the margins of the oval and round windows and may even produce multiple radiolucent foci. Extension of the disease to the otic capsule may produce imaging signs of both fenestral and cochlear otosclerosis in the same patient. Cochlear otosclerosis is characterized by low-attenuation foci around the basal turn of the cochlea, though some lesions may also involve the lateral walls of the IAC and the cochlear promontory. In severe otosclerosis, a low-attenuation ring sometimes surrounds the cochlea, known as the double-ring sign. In the later healing phase, CT may show a "heaped-up" appearance along the margins of the oval and round windows and around the cochlea, indicating new bone formation (Figs 3 and 4). MR imaging is less sensitive for these findings, though T2-weighted images may show a high intraosseous signal intensity in the cochlear form of otosclerosis. ${ }^{6}$

Paget Disease. Paget disease of the temporal bone can rarely cause pulsatile tinnitus. ${ }^{27}$ As in otosclerosis, the development of intraosseous arteriovenous shunts may be the contributing factor. However, unlike otosclerosis, Paget disease is characterized by diffuse involvement of the skull base, producing a cotton-wool appearance (Fig 5).

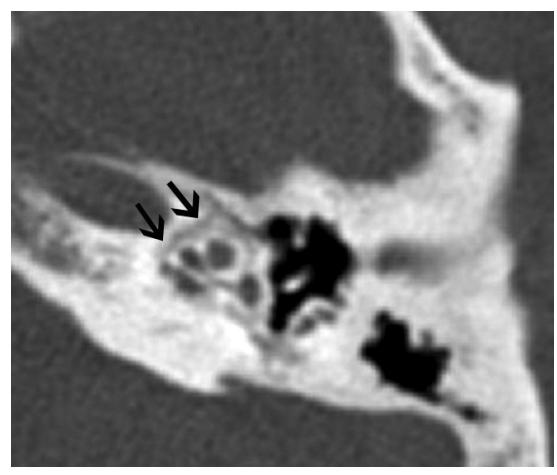

Fig 4. A 50-year-old man with cochlear otospongiosis. Axial CT scan demonstrates abnormal lucency surrounding the left cochlea (black arrows).

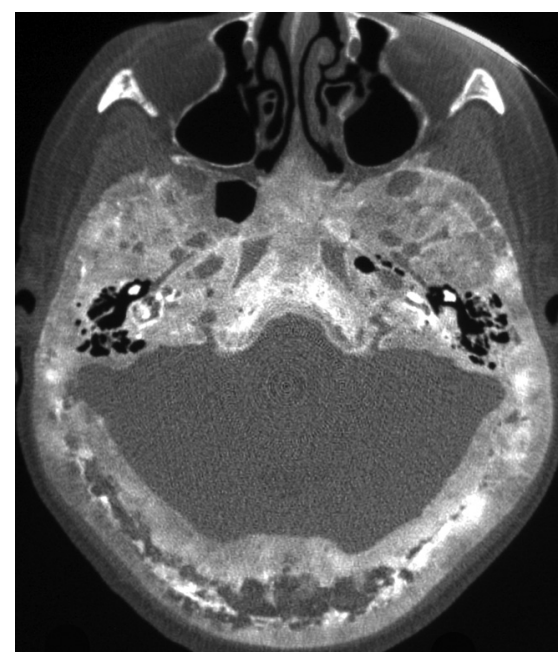

Fig 5. Paget disease of the skull base in a 70-year-old man. Axial CT scan demonstrates diffuse expansion and sclerosis of the bones of the skull base, characteristic of Paget disease. Note the sparing of the maxillofacial bones, which, along with the age of the patient, is a helpful feature in differentiating it from fibrous dysplasia.

Temporal Bone Meningioma. Intraosseous meningiomas of the temporal bone or skull base can occasionally present with tinnitus ${ }^{28}$ and mimic sclerotic fibrous dysplasia on imaging. CT characteristically demonstrates thickening of the teg- 

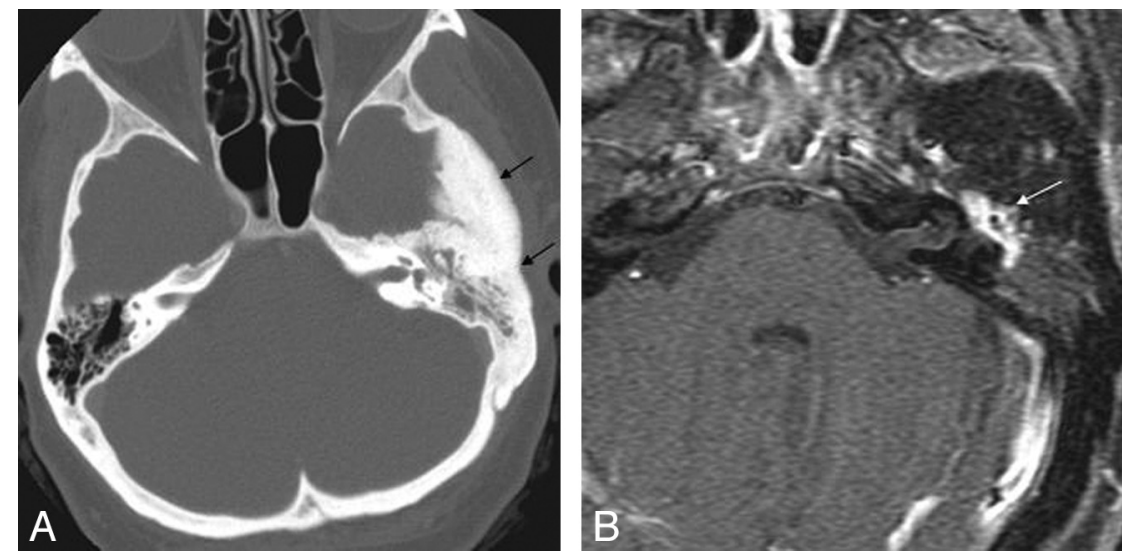

Fig 6. Intraosseous temporal bone meningioma in a 45-year-old woman who presented with left-sided hearing loss and tinnitus. $A$, Axial CT image demonstrates diffuse sclerosis of the temporal bone and soft-tissue attenuation (black arrows) in the left mastoid and middle ear cavity. $B$, Axial enhanced MR image in the same patient demonstrates enhancement within the left middle ear (white arrow).

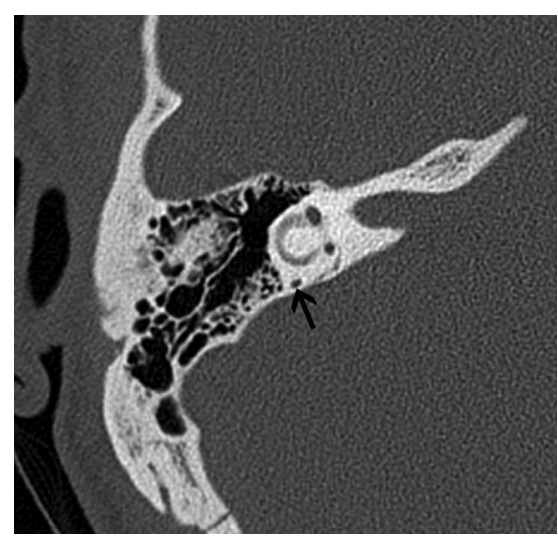

Fig 7. A 50-year-old man with MD. Axial thin-section CT image shows decreased distance between the vertical limb of the posterior semicircular canal and the posterior edge of the temporal bone (black arrow).

men tympani, but the internal trabecular architecture of the involved bone is preserved (Fig 6A). This feature has been termed "trabecular" hyperostosis to distinguish it from the bone thickening observed in cortical hyperostosis or fibroosseous lesions. Characteristic MR imaging findings include en plaque linear dural enhancement along the floor of the middle cranial fossa and homogeneous soft-tissue enhancement (Fig 6B). ${ }^{29}$

\section{Membranous Labyrinth}

MD (Endolymphatic Hydrops). Compared with normal controls, the endolymphatic duct and sac may be difficult to visualize on high-resolution T2-weighted imaging in MD. ${ }^{30-32}$ The gap between the vertical part of the posterior semicircular canal and the posterior fossa may also be narrower than that in healthy subjects ( $2.9 \mathrm{~mm}$ in MD versus $3.8 \mathrm{~mm}$ in controls) (Fig 7). ${ }^{33}$ Patients with MD usually have high-intensity tinnitus, the severity of which may increase with longer duration of the disease; a higher MD stage; and the presence of bilateral disease, hearing impairment, or hyperacusis. ${ }^{34}$

\section{Skull Base Foramina/Canals}

Absence of the carotid canal and enlargement of the inferior tympanic canaliculus are often associated with an aberrant ICA. These signs should trigger a careful examination of the ICA through the middle ear to confirm the diagnosis (as discussed in "Middle Ear").
PSA. Absence of the foramen spinosum and enlargement of the proximal tympanic facial nerve canal may indicate the presence of a PSA (Fig $8 A-C$ ). The stapedial artery is normally present in the fetus but undergoes regression before birth. It arises from a normal or aberrant ICA (approximately 30\% of all aberrant ICAs are associated with a PSA). The PSA typically runs through the obturator foramen (the space between the crura of the stapes) and across the promontory in the middle ear, where it can sometimes be identified. The PSA then runs along the tympanic portion of the facial nerve canal near the geniculate fossa, finally exiting the facial nerve canal to enter the middle cranial fossa to become the middle meningeal artery.

When the stapedial artery persists beyond the fetal period, the middle meningeal artery does not develop from the internal maxillary artery and the foramen spinosum remains undeveloped. The absence of the foramen spinosum is an important CT finding that should alert the radiologist to a possible PSA (Fig 8A). However, these findings do need to be interpreted cautiously because the foramen spinosum may be absent in $\leq 3 \%$ of all skull base CT studies. ${ }^{35}$ The presence of additional signs such as a subtle enlargement of the tympanic segment of the ipsilateral facial nerve canal on coronal CT images, just inferior to the lateral semicircular canal, may raise the index of suspicion (Fig 8B), and DSA can be used for confirmation (Fig 8C). Most patients with a PSA remain asymptomatic and only rarely present with pulsatile tinnitus. The physical examination in some patients may show a red retrotympanic mass, which may provide useful clinical correlation for the imaging findings.

\section{Jugular Foramen}

The differential diagnosis of masses at the jugular foramen includes glomus jugulare or jugulotympanicum, schwannoma, meningioma, metastasis, and pseudomasses due to asymmetric jugular bulb prominence. Of these, the glomus jugulare and jugulotympanicum are most likely to present with pulsatile tinnitus. Rarely, some of the other masses may also cause tinnitus by compressing the jugular vein. ${ }^{36}$

Glomus Jugulare or Jugulotympanicum. Glomus jugulare paragangliomas arise in the jugular bulb and can aggressively erode the bone of the lateral jugular bulb to extend to- 

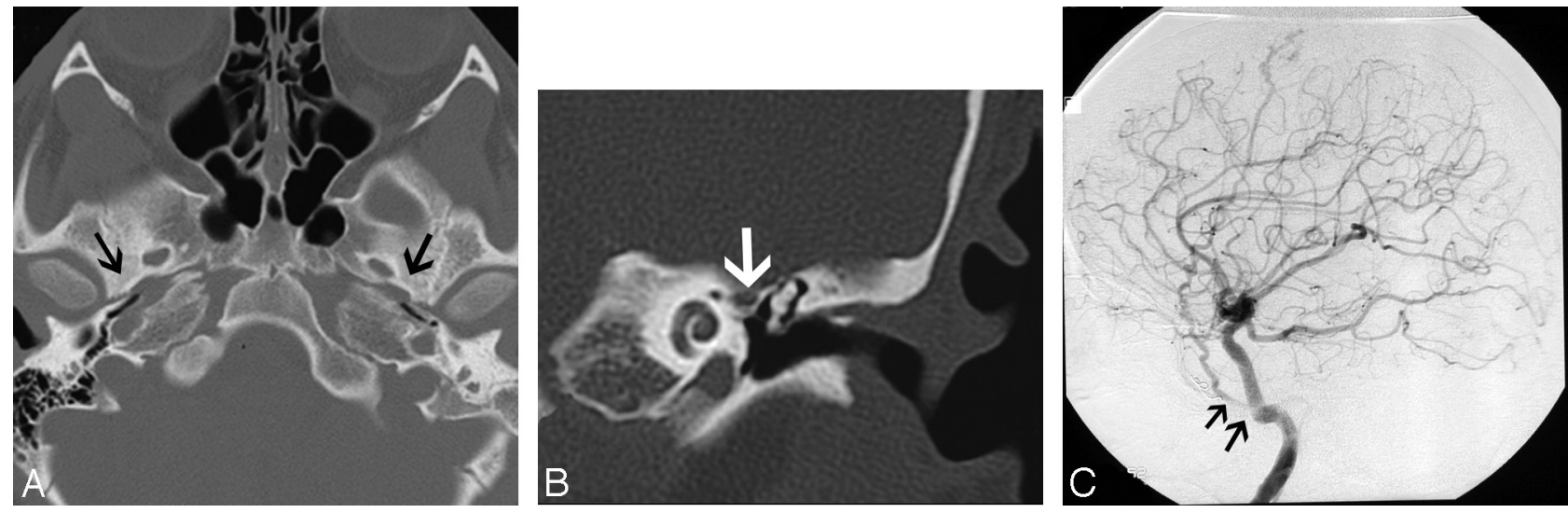

Fig 8. A 30-year-old man with PSA. A, Axial CT image demonstrates absence of the foramen spinosum bilaterally (black arrows). $B$, Coronal CT image in the same patient shows enlargement of the tympanic part of the facial nerve canal, another indirect imaging sign of PSA. C, Lateral DSA image of the internal carotid injection shows the PSA arising from the ICA (arrows).
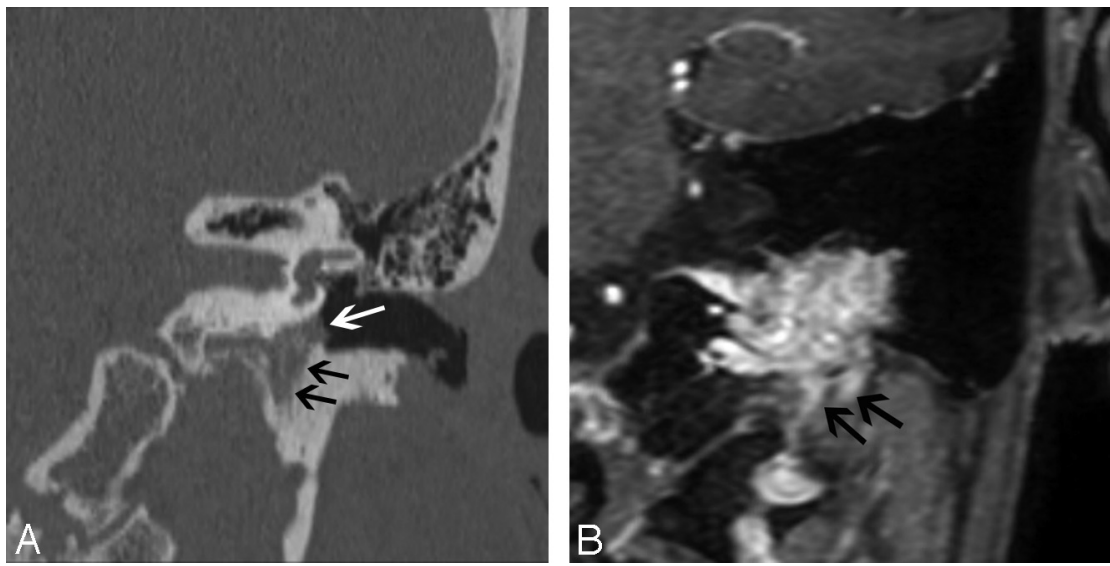

Fig 9. Glomus jugulotympanicum in 45-year-old woman who presented with left-sided pulsatile tinnitus. $A$, Coronal CT image demonstrates soft-tissue attenuation projecting in the hypotympanum (white arrow). There is permeative osseous destruction in the left jugular foramen (black arrows), providing a clue as to the origin of the mass in the left jugular foramen. $B$, Enhanced coronal MR image better demonstrates the true extent of the mass within the left jugular foramen (black arrows).

ward the middle ear. On CT, the presence of permeative bone erosion of the superolateral jugular foramen wall is an important diagnostic clue (Fig 9A). Jugular spine erosion is also common. On MR imaging, large flow voids interspersed with high-signal-intensity parenchyma provide a characteristic "salt and pepper" appearance. Enhanced MR imaging may provide a better estimate of the extent of the mass (Fig 9B). Angiography shows enlarged feeding vessels, a prolonged and intense vascular blush, and early draining veins from arteriovenous shunting.

Glomus tumors should be differentiated from other neoplasms such as meningiomas, schwannomas, and metastases. ${ }^{29}$ Jugular foramen meningiomas are characterized by the presence of sclerotic changes on CT and the absence of flow voids on MR imaging, which contrast with the CT changes of destruction and MR imaging flow voids in jugular paragangliomas. ${ }^{37}$ Similarly, schwannomas appear as sharply demarcated smooth enlargements of the jugular foramen on CT and as contrast-enhancing T2-hyperintense lesions on MR imaging. Unlike glomus tumors that spread superolaterally into the middle ear cavity, jugular foramen schwannomas usually extend superomedially toward the brain stem along lower cranial nerves. ${ }^{37,38}$ Metastases are usually seen as destructive lesions with infiltrative margins on CT and show mixed signal intensity with enhancement on MR imaging. Rarely, lesions such as squamous cell carcinoma, plasmacytoma, chondrosarcoma, Langerhans cell histiocytosis, and lymphoma may also be seen at the jugular foramen. ${ }^{39}$

\section{IAC/CPA}

Vestibular schwannomas are the most frequent CPA masses causing tinnitus. Although the pathophysiology of tinnitus in these patients is not well understood, compression of the cochlear nerve or of its arterial supply is known to cause sensorineural hearing loss and is presumably the cause of the tinnitus as well. Other enhancing CPA masses such as meningiomas, facial or trigeminal nerve schwannomas, and exophytic brain stem gliomas are less likely to present with tinnitus.

Aberrant Anterior Inferior Cerebellar Artery Loops. Vascular loops in contact with the vestibulocochlear nerve are considered to be a normal anatomic variant (Fig 10). However, subjects with pulsatile tinnitus have an 80-fold higher chance of having a contacting vascular loop than patients with nonpulsatile tinnitus. ${ }^{40,41}$ The pathophysiologic role of these loops in tinnitus is unclear.

IAC Hemangiomas. Cavernous angiomas of the IAC are rare causes of tinnitus and hearing loss and can mimic vestibular schwannomas. However, unlike vestibular schwannomas, 


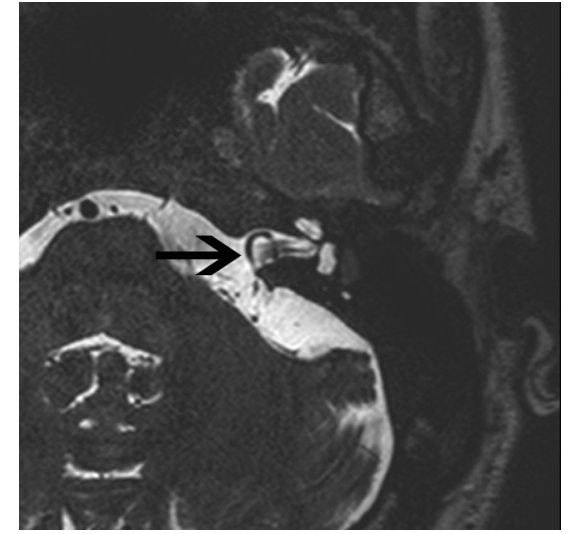

Fig 10. Anterior inferior cerebellar artery loop in a 35-year-old man. Axial T2-weighted MR image shows a vascular loop entering the IAC (black arrow).

IAC hemangiomas frequently cause facial nerve dysfunction even at small tumor sizes. ${ }^{23}$ On MR imaging, these lesions appear intensely enhancing and may be difficult to distinguish from a schwannoma or a meningioma. The detection of calcification/ossification on CT favors a cavernous hemangioma over a schwannoma or a meningioma. ${ }^{42}$

\section{Brain/Intracranial}

Brain Stem Disease. Abnormal brain stem responses to auditory stimuli may be responsible for some forms of continuous tinnitus. ${ }^{4}$ Brain stem diseases such as advanced microvascular disease from hypertension, diabetes or hypercholesterolemia, brain stem strokes, and demyelinating diseases like multiple sclerosis may be implicated. ${ }^{43}$

Palatal myoclonus, a cause of muscular tinnitus, is myoclonus of the tensor and levator veli palatini, tensor tympani, salpingopharyngeus, and the superior constrictor muscles. Cerebellar and brain stem diseases (multiple sclerosis, infarcts) cause palatal myoclonus. ${ }^{44}$ Palatal myoclonus may also occur as an isolated nonprogressive abnormality. Middle ear myoclonus, the other cause of muscular tinnitus, results from rapid rhythmic contractions of the stapedius and tensor tympani muscles and does not have specific imaging correlates.

Chiari I Malformation. Chiari I malformation is rarely associated with tinnitus. Tinnitus in Chiari I malformation is believed to be due to stretching of the eighth nerve complex. ${ }^{45}$

IIH or Pseudotumor Cerebri. IIH has been reported to be the most frequent diagnosis in patients with pulsatile tinnitus. ${ }^{46}$ Imaging findings may be normal, but studies are useful to exclude other causes of increased intracranial pressure. An elevated opening pressure at lumbar puncture confirms the diagnosis.

MR imaging findings of posterior globe flattening, optic nerve sheath distension, optic nerve tortuosity, pituitary deformity, and empty sella turcica are significantly associated with IIH (Fig 11A). However, cross-sectional imaging is neither sensitive nor specific for making this diagnosis. Flattening of the posterior aspect of the globe is the only sign that, if present, is suggestive of the diagnosis of IIH (specificity 100\%, sensitivity $43.5 \%) .{ }^{47}$

Dural Sinus Stenosis. The intracranial dural venous sinuses vary in size in different patients, and often paired sinuses, especially the transverse sinuses, are asymmetric. How- ever, most patients have no related symptoms. If a patient has a focal stenosis of a dural venous sinus, turbulent flow may cause tinnitus. ${ }^{48}$ This abnormality can be assessed with MR imaging (Fig $11 B$ ) or CT venography. MR imaging may also show other signs of intracranial hypertension. The causal relationship of venous sinus stenosis and intracranial hypertension is controversial. ${ }^{49}$ Dilation and stent placement of the lateral venous sinuses have produced clinical improvement in some patients with $\mathrm{IIH}$ who had high venous sinus pressures. $^{50}$

AVM. AVM may be extracranial, dural, or parenchymal, and any of these varieties may be responsible for tinnitus. ${ }^{51,52}$ Signs on CT or MR imaging include the presence of flow voids in the brain parenchyma, evidence of prior hemorrhage on gradient refocused-echo images, abnormal flow patterns, and flow voids in dural venous sinuses, which appear enlarged. However, these findings are not present in most cases, and DSA is the technique of choice whenever an AVM is suspected.

Dural AVF. Dural AVMs or AVFs are the most frequent cause of objective pulsatile tinnitus in the patient with normal findings on otoscopic examination. ${ }^{6}$ In contrast to AVMs that have a nidus composed of a tangle of vessels, dural AVFs usually have a direct communication between an artery and a vein without an intervening nidus. The transverse, sigmoid, and cavernous sinuses are the most frequent locations of dural AVMs and AVFs. Branches of the external carotid artery supply these dural AVMs; venous drainage may be extracranial, intracranial, or both. Conventional angiography is the most sensitive imaging tool.

MR imaging findings of AVMs may include flow-void clusters, engorged ophthalmic vein/proptosis, white matter hyperintensities due to gliosis, intracranial hemorrhages, dilated leptomeningeal or medullary vessels, venous pouches, and leptomeningeal or medullary vascular enhancement. MRA findings may include an identifiable fistula, the presence of a venous flow-related enhancement, and the prominence of extracranial vessels (Fig 12A); DSA demonstrates the lesion best (Fig 12B). ${ }^{7,53}$

\section{Neck}

Carotid Artery Dissection or Stenosis. The turbulent flow that arises from a carotid dissection or atherosclerotic stenosis may cause tinnitus. ${ }^{54} \mathrm{CT}$ angiography may show a typical intimal flap within the arterial lumen. MR imaging is more sensitive for dissection than CT, and unenhanced fat-suppressed T1-weighted images can optimally demonstrate a hyperintense thrombus in the false lumen. A periluminal crescentic focus of high signal intensity ("fried-egg" sign) on axial images is characteristic of carotid dissection.

Fibromuscular dyplasia is an idiopathic inflammatory angiopathy affecting medium-sized vessels. ICAs are the second most common arteries affected; the renal arteries are the most common. Patients most frequently present with brain ischemia, but tinnitus is the next most frequent manifestation. ${ }^{55,56}$ CT or MR arteriography can confirm the diagnosis. Conventional angiography is helpful if angioplasty is being considered. The arteries have a characteristic beaded appearance from multifocal stenoses separated by regions of variable dilation. 

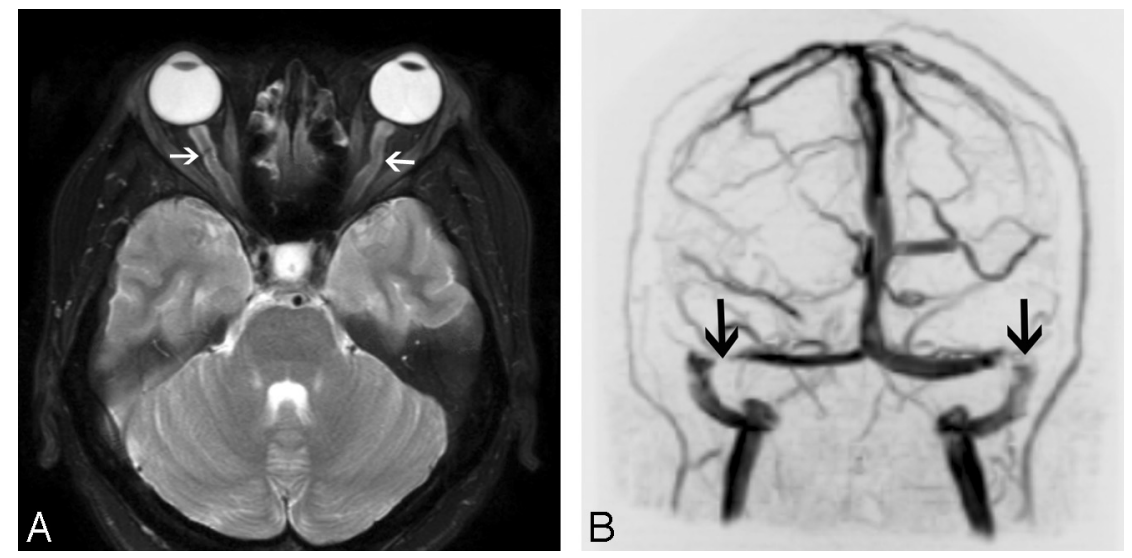

Fig 11. Intracranial hypertension in a 30-year-old woman presenting with headaches and tinnitus. A, Axial T2-weighted image demonstrates dilation of bilateral optic nerve sheaths (arrows) and flattening of the posterior globes. B, Coronal maximum-intensity-projection image from an MR venogram also demonstrates stenoses of bilateral transverse sinuses (arrows).
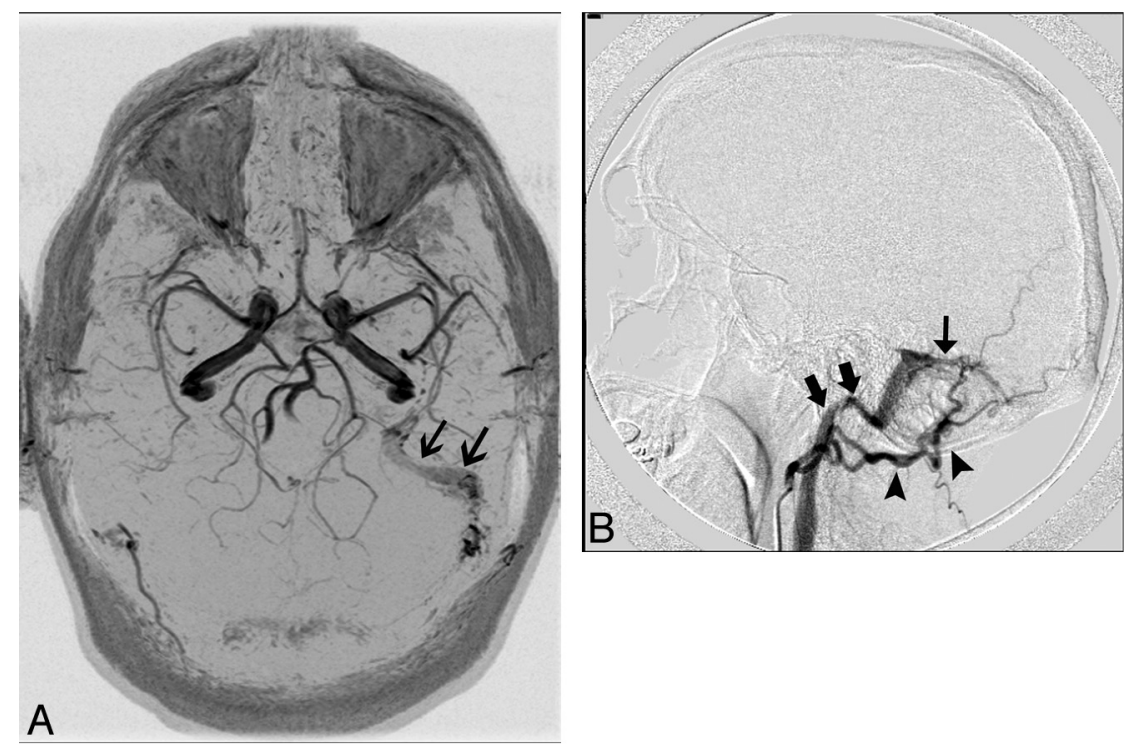

Fig 12. A 40-year-old man who presented with left-sided pulsatile tinnitus. A, MR angiogram demonstrates asymmetric increased flow-related enhancement in the left sigmoid sinus (black arrows) and multiple small arterial flow voids within it. B, Lateral projection of DSA shows injection of the left occipital artery (arrowheads) opacifying the AVF (thin black arrow) and rapid opacification of the left sigmoid sinus and internal jugular vein (thick black arrows).

Venous Obstruction. Although venous hums are common in asymptomatic persons, ${ }^{57}$ the compression of the jugular vein in the neck or mediastinal venous compression may cause tinnitus due to turbulent blood flow. When a detailed CT or MR imaging study of the temporal bone fails to disclose a cause for pulsatile tinnitus in a patient, it may be useful to image the neck and the superior mediastinum to search for a mass compressing the internal jugular vein anywhere along its course.

\section{TMJ}

TMJ degeneration is often overlooked as a potential cause of head and neck symptoms. Patients with degenerative disease of the TMJ may present with continuous tinnitus. ${ }^{58,59}$ The mechanism for this relationship is not well understood. The tension in the pterygoid muscles, which are attached to the $\mathrm{TMJ}$, may increase tension in the tensor tympani, possibly mediated by their common innervation through the mandibular division of the trigeminal nerve. MR imaging is the preferred technique for TMJ assessment.

\section{Conclusions}

Many patients will have no radiologic abnormalities than can explain their tinnitus. On the other hand, many radiologic findings associated with tinnitus are also seen frequently in asymptomatic individuals. The correlation of imaging findings with clinical symptoms remains a critical step before suggesting that a particular radiologic finding may be responsible for a patient's tinnitus. A compartment-based approach in the imaging evaluation helps to analyze systematically the various imaging modalities, which will prevent missing any important finding that may be a potential cause for tinnitus.

\section{Acknowledgment}

The authors acknowledge Ms. Pat Moore, Information System Specialist, Radiology Administration Section, University of Alabama at Birmingham for her help with the illustrations.

\section{References}

1. Meyerhoff WL, Cooper JC. Tinnitus. In: Paparella MM, ed. Otolaryngology. 3rd ed. Philadelphia: Saunders; 1991:1169-75 
2. Schleuning AJ 2nd. Management of the patient with tinnitus. Med Clin North Am 1991;75:1225-37

3. Lockwood AH, Salvi RJ, Burkard RF. Tinnitus. N Engl J Med 2002;347:904-10

4. Bauer CA. Mechanisms of tinnitus generation. Curr Opin Otolaryngol Head Neck Surg 2004;12:413-17

5. Sismanis A. Tinnitus. Curr Neurol Neurosci Rep 2001;1:492-99

6. Remley KB, Coit WE, Harnsberger HR, et al. Pulsatile tinnitus and the vascular tympanic membrane: CT, MR, and angiographic findings. Radiology 1990;174: 383-89

7. Dietz RR, Davis WL, Harnsberger HR, et al. MR imaging and MR angiography in the evaluation of pulsatile tinnitus. AJNR Am J Neuroradiol 1994;15:879-89

8. Sonmez G, Basekim CC, Ozturk E, et al. Imaging of pulsatile tinnitus: a review of 74 patients. Clin Imaging 2007;31:102-08

9. Waldvogel D, Mattle HP, Sturzenegger M, et al. Pulsatile tinnitus: a review of 84 patients. J Neurol 1998;245:137-42

10. Sismanis A, Smoker WR. Pulsatile tinnitus: recent advances in diagnosis. $\mathrm{La}$ ryngoscope 1994;104:681-88

11. Willinsky RA. Tinnitus: imaging algorithms. Can Assoc Radiol J 1992;43:93-99

12. Weissman JL, Hirsch BE. Imaging of tinnitus: a review. Radiology 2000;216: 342-49

13. Shin EJ, Lalwani AK, Dowd CF. Role of angiography in the evaluation of patients with pulsatile tinnitus. Laryngoscope 2000;110:1916-20

14. Cashen TA, Carr JC, Shin W, et al. Intracranial time-resolved contrast-enhanced MR angiography at 3T. AJNR Am J Neuroradiol 2006;27:822-29

15. Zou Z, Ma L, Cheng L, et al. Time-resolved contrast-enhanced MR angiography of intracranial lesions. J Magn Reson Imaging 2008;27:692-99

16. Khodadad G. Arteriovenous malformations of the scalp. Ann Surg 1973;177: 79-85

17. Courteney-Harris RG, Ford GR, Innes AJ, et al. Pulsatile tinnitus: three cases of arteriovenous fistula treated by ligation of the occipital artery. J Laryngol Otol 1990;104:421-22

18. Weissman JL, Hirsch BE. Beyond the promontory: the multifocal origin of glomus tympanicum tumors. AJNR Am J Neuroradiol 1998;19:119-22

19. Lo WW, Solti-Bohman LG, McElveen JT Jr. Aberrant carotid artery: radiologic diagnosis with emphasis on high-resolution computed tomography. Radiographics 1985;5:985-93

20. Davis WL, Harnsberger HR. MR angiography of an aberrant internal carotid artery. AJNR Am J Neuroradiol 1991;12:1225

21. Koesling S, Kunkel P, Schul T. Vascular anomalies, sutures and small canals of the temporal bone on axial CT. Eur J Radiol 2005;54:335-43

22. Tokyol C, Yilmaz MD. Middle ear hemangioma: a case report. Am JOtolaryngol 2003;24:405-07

23. Lo WW, Shelton C, Waluch V, et al. Intratemporal vascular tumors: detection with CT and MR imaging. Radiology 1989;171:445-48

24. Isaacson B, Telian SA, McKeever PE, et al. Hemangiomas of the geniculate ganglion. Otol Neurotol 2005;26:796-802

25. Friedman $\mathrm{O}, \mathrm{Neff} \mathrm{BA}$, Willcox TO, et al. Temporal bone hemangiomas involving the facial nerve. Otol Neurotol 2002;23:760-66

26. Gristwood RE, Venables WN. Otosclerosis and chronic tinnitus. Ann Otol Rhinol Laryngol 2003;112:398-403

27. Mackenzie I, Young C, Fraser WD. Tinnitus and Paget's disease of bone. $J$ Laryngol Otol 2006;120:899-902. Epub 2006 Sep 29

28. Prayson RA. Middle ear meningiomas. Ann Diagn Pathol 2000;4:149-53

29. Hamilton BE, Salzman KL, Patel N, et al. Imaging and clinical characteristics of temporal bone meningioma. AJNR Am J Neuroradiol 2006;27:2204-09

30. Lorenzi MC, Bento RF, Daniel MM, et al. Magnetic resonance imaging of the temporal bone in patients with Meniere's disease. Acta Otolaryngol 2000;120: 615-19

31. Naganawa S, Asai $H$, Ishigaki $T$, et al. MR imaging of the vestibular aqueduct in normal volunteers and patients with Meniere's disease: a preliminary report. Nippon Igaku Hoshasen Gakkai Zasshi 1991;51:213-18

32. Tanioka $\mathrm{H}$, Zusho $\mathrm{H}$, Machida $\mathrm{T}$, et al. High-resolution MR imaging of the inner ear: findings in Meniere's disease. Eur J Radiol 1992;15:83-88

33. Mateijsen DJ, Van Hengel PW, Krikke AP, et al. Three-dimensional Fourier transformation constructive interference in steady state magnetic resonance imaging of the inner ear in patients with unilateral and bilateral Meniere's disease. Otol Neurotol 2002;23:208-13

34. Herraiz C, Tapia MC, Plaza G. Tinnitus and Meniere's disease: characteristics and prognosis in a tinnitus clinic sample. Eur Arch Otorhinolaryngol 2006;263: 504-09

35. Ginsberg LE, Pruett SW, Chen MY, et al. Skull-base foramina of the middle cranial fossa: reassessment of normal variation with high-resolution CT. AJNR Am J Neuroradiol 1994;15:283-91

36. Sharma RR, Pawar SJ, Dev E, et al. Vagal schwannoma of the cerebello-medullary cistern presenting with hoarseness and intractable tinnitus: a rare case of intra-operative bradycardia and cardiac asystole. J Clin Neurosci 2001;8: 577-80

37. Macdonald AJ, Salzman KL, Harnsberger HR, et al. Primary jugular foramen meningioma: imaging appearance and differentiating features. $A J R A m$ Roentgenol 2004;182:373-77

38. Eldevik OP, Gabrielsen TO, Jacobsen EA. Imaging findings in schwannomas of the jugular foramen. AJNR Am J Neuroradiol 2000;21:1139-44

39. Caldemeyer KS, Mathews VP, Azzarelli B, et al. The jugular foramen: a review of anatomy, masses, and imaging characteristics. Radiographics 1997;17: 1123-39

40. Nowe V, De Ridder D, Van de Heyning PH, et al. Does the location of a vascular loop in the cerebellopontine angle explain pulsatile and non-pulsatile tinnitus? Eur Radiol 2004;14:2282-89

41. Chadha NK, Weiner GM. Vascular loops causing otological symptoms: a systematic review and meta-analysis. Clin Otolaryngol 2008;33:5-11

42. Omojola MF, al Hawashim NS, Zuwayed MA, et al. CT and MRI features of cavernous haemangioma of internal auditory canal. Br J Radiol 1997;70: $1184-87$

43. Branstetter BF, Weissman JL. The radiologic evaluation of tinnitus. Eur Radiol 2006;16:2792-802

44. Tyler RS, Babin RW. Tinnitus. In: Cummings CW, Fredrickson JM, Harker LA et al, eds. Otolaryngology-Head and Neck Surgery. 2nd ed. St. Louis: Mosby-Year Book; 1993:3031-53

45. Weber PC, Cass SP. Neurotologic manifestations of Chiari 1 malformation. Otolaryngol Head Neck Surg 1993;109:853-60

46. Sismanis A. Pulsatile tinnitus: a 15-year experience. Am J Otol 1998;19:472-77

47. Agid R, Farb RI, Willinsky RA, et al. Idiopathic intracranial hypertension: the validity of cross-sectional neuroimaging signs. Neuroradiology 2006;48: 521-27

48. Mathis JM, Mattox D, Malloy P, et al. Endovascular treatment of pulsatile tinnitus caused by dural sinus stenosis. Skull Base Surg 1997;7:145-50

49. Ball AK, Clarke CE. Idiopathic intracranial hypertension. Lancet Neurol 2006;5:433-42

50. Higgins JN, Owler BK, Cousins C, et al. Venous sinus stenting for refractory benign intracranial hypertension. Lancet 2002;359:228-30

51. Shah SB, Lalwani AK, Dowd CF. Transverse/sigmoid sinus dural arteriovenous fistulas presenting as pulsatile tinnitus. Laryngoscope 1999;109:54-58

52. Roy D, Lavigne F, Raymond J. Pulsatile tinnitus and dural arteriovenous fistula of the transverse sinus. J Otolaryngol 1993;22:409-12

53. Kwon BJ, Han MH, Kang HS, et al. MR imaging findings of intracranial dural arteriovenous fistulas: relations with venous drainage patterns. AJNR Am J Neuroradiol 2005;26:2500-07

54. Vories A, Liening D. Spontaneous dissection of the internal carotid artery presenting with pulsatile tinnitus. Am J Otolaryngol 1998;19:213-15

55. Dufour JJ, Lavigne F, Plante R, et al. Pulsatile tinnitus and fibromuscular dysplasia of the internal carotid. J Otolaryngol 1985;14:293-95

56. Foyt D, Carfrae MJ, Rapoport R. Fibromuscular dysplasia of the internal carotid artery causing pulsatile tinnitus. Otolaryngol Head Neck Surg 2006;134: 701-02

57. Nehru VI, al-Khaboori MJ, Kishore K. Ligation of the internal jugular vein in venous hum tinnitus. J Laryngol Otol 1993;107:1037-38

58. Parker WS, Chole RA. Tinnitus, vertigo, and temporomandibular disorders. Am J Orthod Dentofacial Orthop 1995;107:153-58

59. Chole RA, Parker WS. Tinnitus and vertigo in patients with temporomandibular disorder. Arch Otolaryngol Head Neck Surg 1992;118:817-21 